\title{
Perfil do nível de atividade física de idosos hipertensos e diabéticos
}

\author{
Viviane Rech", Hugo Tourinho Filho**, Maria Manuela Martins ${ }^{* * *}$
}

\section{Resumo}

O estudo teve como objetivo verificar o nível de habitual atividade física em grupo de convivência de idosos, caracterizando-se como descritivo de corte transversal. A amostra constituiu-se de 1.919 idosos (1.705 mulheres e 214 homens) com média de idade 68,73 $\pm 6,99$ anos e com hipertensão arterial e diabetes mellitus associadas. Os idosos foram convidados a, voluntariamente, responder o questionário semiestruturado IPAq e o tempo de prática de atividade física mais prevalente (ginástica, dança, caminhada, atividades em circuito e exercícios de fortalecimento de membros superiores e inferiores, alongamentos, além de atividades leves como as domiciliares ou de transporte). O tempo de prática foi aferido por meio de questionário semiestruturado. Os dados foram analisados por meio do programa estatístico SPSS ${ }^{\circledR}$ 17.0, utilizando-se de análises descritivas. No que se refere à prática de atividade física habitual, os idosos, na maioria $(71 \%)$, foram considerados ativos. $\mathrm{Na}$ contribuição dos diferentes domínios da atividade física, quanto ao dispêndio total em minutos por semana de atividades físicas, destacam-se com maior prevalência as atividades de lazer/recreação (38\% e $33 \%$ ), respectivamente, com uma frequência de duas vezes por semana por um tempo de $1 \mathrm{~h} 30 \mathrm{~min}$ e atividades no domicílio (21\%). Portanto, os idosos participantes apresentaram-se ativos, principalmente em atividades de lazer/recreação, seguidas das atividades do lar, e menos ativos nos domínios trabalho e transporte. Sugere-se que mais estudos sejam efetuados com esse mesmo enfoque, a fim de verificar, no longo prazo, os níveis de atividades físicas dessa população, como um acompanhamsento periódico.

Palavras-chave: Idosos. Atividade física. Prevalência. Hipertensão arterial. Diabetes.

* Fisioterapeuta graduada pela Universidade de Cruz Alta (Unicruz). Especialista em Atividade Fsica e Qualidade de Vida pela Universidade de Passo Fundo (UPF). Mestra em Tecnologia em Saúde pela Pontifícia Universidade Católica do Paraná (PUCPR). Doutoranda em Ciências do Desporto na Universidade de Trás-os-Montes e Alto Douro (UTAD). Endereço para correspondência: Rua Dom Sebastião Lemos, 115, Bairro Santa Teresinha, CEP: 99074-33, Passo Fundo - RS. E-mail: respvi@gmail.com.

** Graduado em Educação Física pela Universidade Estadual de Londrina (UEL). Especialista em Avaliação da Performance Motora - Universidade Estadual de Londrina (UEL). Mestre em Ciência do Movimento Humano pela Universidade Federal de Santa Maria (UFSM). Doutor em Educação Física pela Universidade de São Paulo (USP). Docente da Escola de Educação Física e Esporte da Universidade de São Paulo (EEFERP/USP) - Campus Ribeirão Preto.

**** Docente da Escola Superior de Enfermagem do Porto - Porto - ESENF - Portugal. Enfermeira graduada pela Escola de Enfermagem Calouste Gulbenkian, em Braga. Especialista em Enfermagem de Reabilitação pela Escola de Enfermagem Pós-Básica no Porto. Especialista em Administração dos Serviços de Enfermagem pela Escola Superior de Enfermagem Cidade do Porto. Mestra em Planificacion, Disenõ e Investigation em Serviços Sociales, da Universidade da Estremadura, equivalência ao grau de mestrado em Ciências de Enfermagem na Universidade do Porto - Instituto de Ciências Biomédicas Abel Salazar. Doutora em Ciências de Enfermagem pela Universidade do Porto - Instituto de Ciências Biomédicas Abel Salazar.

$\hookrightarrow$ doi:10.5335/rbceh.2012.040 


\section{Introdução}

Do ponto de vista demográfico, o envelhecimento é caracterizado pelo aumento na proporção da população a partir de 60 anos para países em desenvolvimento e de 65 anos para os desenvolvidos, em relação à população total. (CARVALHO; GARCIA, 2003).

$\mathrm{O}$ envelhecer evidencia-se muito nas sociedades atuais. Documentado na literatura científica, Spirduso (2005), Shephard (1991) e IBGE (2002) sugerem que o processo de envelhecimento está associado a alterações físicas, fisiológicas, psicológicas e sociais, bem como ao surgimento de enfermidades crônicas advindas de hábitos de vida não adequados, tais como tabagismo, alimentação incorreta, tipo de atividades diárias, ausência de atividades físicas regulares, que se refletem na redução da capacidade para realização das atividades de vida diária.

Em relação ao perfil epidemiológico das doenças, na população idosa, predominam as chamadas doenças crônicas não transmissíveis (DCNT), que são representadas pelas doenças cardiovasculares, sequelas de doenças cerebroarticulares, osteoarticulares, diabetes mellitus (DM), doenças pulmonares e doenças psicorgânicas, como os diversos tipos de demência. No que diz respeito aos fatores de risco, Silva (2009) chama a atenção para um fator importante para a população idosa, que é a história social, a qual tem um papel determinante nas patologias do idoso. Sabe-se que existem diversas particularidades que caracterizam o processo de envelhecimento como alterações estruturais e funcionais, das quais se destacam a hipertensão arterial sistêmica (HAS) e o diabetes mellitus tipo II (DMII).

A HAS, doença cardiovascular de alta prevalência mundial, acomete mais de $60 \%$ da população formada por pessoas com 60 anos ou mais, havendo maior envolvimento naqueles da etnia negra e do sexo feminino. (RÊGO, 2011). No Brasil, esse fato se dá de modo bastante acelerado. (OLIVEIRA, 2008).

Outra doença crônica de grandes repercussões na saúde dos idosos é o diabetes mellitus. Essa patologia metabólica silenciosa é caracterizada pelo aumento anormal da glicemia provocada pela falta de insulina, absoluta ou relativa. De acordo com a International Diabetes Federation, o número total de diabéticos tem crescido significativamente, sendo 246 milhões em 2007 e prevendo-se um aumento para 380 milhões em 2025. (SILVA, 2009). Nos países ocidentais, constitui uma das principais responsáveis por doença renal crônica terminal com necessidade de terapêutica dialítica, bem como a principal causa de cegueira na idade adulta entre os 20 e os 74 anos e de amputação não traumática dos membros inferiores. (SILVA, 2009; DUARTE, 2007; PNPCD, 2007; CDTC, 2005).

Nas últimas décadas, houve uma expansão no que tange a programas de exercícios físicos e formação de grupos de convivência para a terceira idade. (BENEDETTI, 2004). Observa-se o fomento de programas de atividades físicas direcionados aos idosos por instituições públicas e de ensino. No entanto, a participação e adesão a esses programas pelo público de destino dependem das características individuais, culturais, sociais, tanto quanto da conscientização dos bene- 
fícios que a atividade física proporciona. (ALONSO; SANTOS; FIGUEIRA, 2006).

As maiores evidências para melhor qualidade de vida aos idosos, diante das DCNT, existem com programas que incluem treinamento de força, sendo o treinamento de alta intensidade mais benéfico e seguro do que o de baixa intensidade. Por isso, todos os programas de exercício para o idoso frágil devem incluir treinamento de resistência progressiva dos grandes grupamentos musculares das extremidades inferiores e superiores e do tronco. Programas de no mínimo duas, mas preferivelmente três vezes por semana, são recomendados, com duas ou três séries de cada exercício desempenhado em cada dia de treinamento. Recomenda-se, também, algumas posturas em pé com pesos livres, utilizadas para incrementar, simultaneamente, o equilíbrio e a coordenação muscular. $\mathrm{O}$ treinamento do equilíbrio pode também ser incorporado como parte do treinamento de força ou como uma modalidade separada. $\mathrm{O}$ treinamento e a supervisão, especialmente para os muito frágeis, são obrigatórios para a segurança e a progressão ocorrerem. (PAPALIA; OLDS, 2000).

São cada vez mais evidenciados os benefícios dos exercícios físicos nessa população, e as pesquisas têm demonstrado sua capacidade de minimizar o processo degenerativo provocado pelo envelhecimento, além da sua contribuição para a qualidade de vida dos indivíduos, influenciando na sua independência funcional. (OKUMA, 2002; PAFFENBARGER, 1994).

Seguindo essa linha de raciocínio, o objetivo desta pesquisa foi verificar o nível de atividade física habitual em idosos hipertensos e diabéticos praticantes de atividades físicas em grupo de convivência.

Neste estudo, foi utilizado o termo "atividade física" para designar qualquer movimento corporal e/ou funcional produzido pelos músculos esqueléticos, resultando em gasto energético maior que os níveis de repouso. (CASPERSEN; POWELL; CHRISTENSON, 2010).

\section{Material e métodos}

A pesquisa caracteriza-se como descritiva de corte transversal e compreende idosos participantes de grupo de vivência que realizam atividades físicas regulares duas vezes por semana, durante 1h30min, em cada dia de encontro, na cidade da pesquisa. Foram admitidos, como critério de inclusão para o estudo, indivíduos com idade igual ou superior a 60 anos, de ambos os sexos, com tempo de participação mínima de seis meses no grupo. As atividades realizadas eram ginástica, dança, caminhada, atividades em circuito e exercícios de fortalecimento de membros superiores e inferiores, além dos alongamentos inicial e final. A amostra constituiu-se de 1.919 idosos simultaneamente hipertensos e diabéticos.

A coleta de dados foi realizada, inicialmente, com a captação, por meio de um questionário básico, de informações referentes à demografia, patologia clínica e escolaridade, sendo este último fator determinante para a compreensão e 0 preenchimento do questionário.

Utilizou-se o Questionário Internacional de Atividades Físicas (IPAq), 
considerado um dos mais indicados para identificar níveis gerais de atividades físicas em idosos. Por meio dele, é mensurado o tempo de atividades físicas realizadas, sendo elas moderadas e/ou vigorosas nos domínios do transporte, trabalho, domicílio e de lazer/recreação (moderados e vigorosos) numa semana cotidiana. Como classificação do nível de atividade física, foram considerados menos ativos os idosos que, no somatório de todos esses domínios, apresentavam menos de 150 minutos de atividades físicas moderadas ou vigorosas durante uma semana, e mais ativos os idosos que obtiveram um nível superior a 150 minutos semanais. (PATE et al., 1995). Realizou-se a mensuração da quantidade de dias (semana normal/ habitual), do tempo (horas e minutos por dia e semana) e da intensidade (leve ou moderada ou vigorosa) na realização das atividades físicas nos diferentes domínios. Como se tratou de um grupo de idosos que já realizava atividades físicas como forma de exercícios regulares para a saúde, foi excluída a velocidade lenta do questionário. Para determinar a porcentagem da amostra que realizava atividade física dentro da recomendação atual (MATSUDO et al., 2001) (pelo menos 30 minutos por dia, na maior parte dos dias da semana), foi calculada para a análise a somatória de pelo menos 150 minutos por semana de atividade física de intensidade pelo menos moderada. Para atividade física vigorosa, o critério foi de pelo menos três sessões de 20 minutos por semana atual. (MATSUDO et al., 2001).

O tempo de prática é uma variável relacionada ao tempo completado em anos a que os idosos praticam atividades físicas regularmente no grupo de convivência selecionado para este estudo. Para tanto, foram considerados como iniciantes, configurando-se em critério de exclusão para a pesquisa, os idosos que estavam começando as atividades no grupo ou os que ainda não haviam completado seis meses de participação nas atividades e na prática de exercícios naquele contexto, independentemente de terem participado de grupos de convivência semelhantes.

Os dados referentes a essa variável foram obtidos por meio de entrevista individual. Entre os procedimentos para a coleta de dados, realizou-se um treinamento para a capacitação na aplicação do IPAq, que serviu para orientar os entrevistadores de forma a homogeneizar as condutas e assegurar uma boa compreensão e entendimento do instrumento, diminuindo, assim, as interpretações equivocadas que pudessem comprometer os resultados. Os idosos assinaram o Termo de Consentimento Livre e Esclarecido, e o projeto de pesquisa foi aprovado pela Secretaria de Saúde do Município e pelo Comitê de Ética e Pesquisa locais, mediante Parecer $n^{\circ}$ 257/2009. A análise estatística foi realizada pelo programa estatístico SPSS ${ }^{\circledR}$ 17.0. Utilizou-se o teste de normalidade Kolmogorov-Smirnov para descrição dos dados em média e desvio padrão. Foram calculadas, também, frequências relativas e absolutas.

\section{Resultados}

Os idosos participantes da pesquisa tinham em média de idade 68,73 $\pm 6,99$ anos, com diagnóstico médico de HAS e DMII. Destes, 1.705 eram mulheres 
e 214 eram homens. Em relação à escolaridade, predominaram idosos com ensino fundamental (43\%). Observa-se, na Tabela 1, que, ao categorizar a atividade física em dois níveis, houve uma predominância dos idosos mais ativos $(81,81 \%)$ sobre os menos ativos
(70,87\%). Possivelmente, os percentuais de idosos mais ativos foram maiores no presente estudo em razão de suas características particulares, ou seja, do fato de se tratar de um grupo específico de convivência que participa de atividades físicas regulares.

Tabela 1 - Resultado dos domínios atividades moderadas e vigorosas, que compõem o IPAq, representados por meio de frequência (n), percentual (\%), média, desvio padrão (DP) e mediana da amostra parcial dos idosos hipertensos/diabéticos.

\begin{tabular}{lcccc}
\hline Características & $\begin{array}{c}(\mathrm{n}=1.919) \\
\text { Parcial }\end{array}$ & $\begin{array}{c}\% \\
\text { Parcial }\end{array}$ & $\begin{array}{c}\text { Média }( \pm \mathrm{DP}) \\
\mathrm{min} / \mathrm{sem}\end{array}$ & $\begin{array}{c}\text { Mediana } \\
\mathrm{min} / \mathrm{sem}\end{array}$ \\
\hline Ativ. moderada & 1.360 & 70,87 & $53,17( \pm 6,16)$ & 30 \\
Ativ. vigorosa & 1.570 & 81,81 & $42,78( \pm 7,47)$ & 17,14 \\
\hline
\end{tabular}

O IPAq tem sido empregado como um dos instrumentos de mensuração do nível de atividade física em grandes grupos populacionais de boa precisão e de baixo custo. Estão apresentados na Tabela 2 os valores em frequência relativa e absoluta, média, desvio padrão e mediana dos diferentes domínios, considerando todos os participantes do estudo ( $\mathrm{n}$ total) e apenas os idosos que realizaram atividade física de intensidade moderada e/ ou vigorosa com duração mínima de 10 minutos contínuos para cada domínio (n parcial).

Tabela 2 - Resultado dos diferentes domínios que compõem o IPAq representados por meio de frequência (n), percentual (\%), média, desvio padrão (DP) e mediana da amostra total dos idosos hipertensos/diabéticos.

\begin{tabular}{ccccc}
\hline Características & $\begin{array}{c}(\mathrm{n}=1.919) \text { Hipertensos/ } \\
\text { diabéticos }\end{array}$ & $\begin{array}{c}\text { \% Hipertensos/ } \\
\text { diabéticos }\end{array}$ & $\begin{array}{c}\text { Média }( \pm \mathrm{DP}) \\
\mathrm{min} / \mathrm{sem}\end{array}$ & $\begin{array}{c}\text { Mediana } \\
\text { min/sem }\end{array}$ \\
\hline $\begin{array}{c}\text { Ativ. moderada } \\
\text { (lazer/recreação) }\end{array}$ & 1.919 & 100 & $53,17( \pm 6,16)$ & 30 \\
$\begin{array}{c}\text { Ativ. vigorosa } \\
\text { (lazer/recreação) }\end{array}$ & 1.919 & 100 & $42,78( \pm 7,47)$ & 17,14 \\
Ativ. no trabalho & 1.919 & 100 & $242,88( \pm 28,18)$ & 154,29 \\
Ativ. no transporte & 1.919 & 100 & $65,58( \pm 11,46)$ & 25,71 \\
Ativ. em casa & 1.919 & 100 & $187,20( \pm 16,08)$ & 154,29 \\
\hline
\end{tabular}


Considerando o total de idosos participantes do estudo ( $\mathrm{n}=1.919)$, a média de atividade física habitual mais significativa está relacionada ao trabalho, remunerado e/ou voluntariado, constatando-se $242,88 \mathrm{~min} / \mathrm{sem}$. A maioria realiza atividades leves, como pintura, costura, bordado, culinária, marcenaria básica, jardinagem e trabalhos artesanais, sendo o domínio a que os idosos dedicam um tempo maior durante uma semana normal/cotidiana.

No domínio do transporte, observou-se que os idosos gastavam $65,58 \pm 11,46$ $\mathrm{min} / \mathrm{sem}$, preferencialmente caminhando ou andando de bicicleta como forma de deslocamento.

Nas atividades físicas realizadas no âmbito doméstico, com duração mínima de 10 minutos contínuos, constatou-se que, em média, 187,20 $\pm 16,08 \mathrm{~min} / \mathrm{sem}$ eram dedicados a essas tarefas, sejam de arrumação da casa, preparo de alimentos, limpeza ou ocupação com animais de estimação.

Para o domínio atividades moderadas, consideradas atividades de lazer/ recreação pelos participantes, apresentou-se a média semanal de $53,17 \pm 6,16$ minutos.

No domínio em que o dispêndio de tempo numa semana normal/usual apresentou uma média semanal de 42,78 \pm 7,47 minutos em atividades vigorosas como ginástica, dança, caminhada, atividades em circuito e exercícios de fortalecimento de membros superiores e inferiores, além dos alongamentos - também considerado de atividades de lazer/ recreação pelos idosos -, constatou-se $81,81 \%$, analisando-se o n separadamente, e, no domínio atividades moderadas, um total de 70,87\% do n parcial. Provavelmente, tal predominância ocorra em razão da característica do grupo em estudo, ou seja, um grupo de convivência em que os idosos participam de forma regular das atividades físicas.

Ao observar-se os cinco domínios que compõem o IPAq (Figura 1), os resultados indicam que o período de tempo ( $\mathrm{min} / \mathrm{sem})$ de atividades moderadas e vigorosas realizadas por pelo menos 10 minutos contínuos apresentou uma predominância das atividades físicas de lazer/recreação (38\% e 33\%), respectivamente, tendo os avaliados considerado como seus únicos momentos da semana de lazer/recreação os que correspondiam à participação no grupo de convivência. Isso foi seguido das atividades físicas no âmbito doméstico (21\%), sendo as mais citadas limpeza e arrumação da casa e do jardim. Nas atividades físicas como meio de transporte (4\%), foram mencionadas pequenas caminhadas até locais de venda de alimentos ou lojas de medicamentos. Mesmo percentual (4\%) obteve a realização de atividades físicas no trabalho (4\%), sendo relatado que, na maioria do tempo, as atividades laborais eram realizadas paradas e com pouco esforço para o manuseio de objetos. 


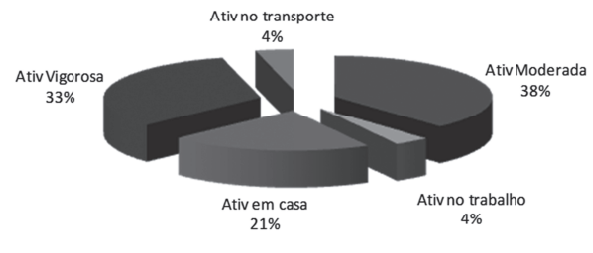

Figura 1 - Valores em percentuais dos diferentes domínios que compõem o IPAq, considerando os idosos que fazem mais de 10 minutos de atividades contínuas.

\section{Discussões}

Em relação aos níveis de atividade física habitual, em estudos que avaliaram idosos residentes em regiões interioranas do estado de Santa Catarina/ Brasil, utilizando o mesmo instrumento para mensurar o nível de atividade física (IPAq) e adotando os mesmos critérios para categorizá-la, foram encontrados os seguintes resultados: considerando 198 idosos portadores de patologias crônico-degenerativas, com a média de idade de 73,6 anos (DP=5,9), participantes em 33 grupos de convivência para idosos, $33,8 \%$ foram identificados como menos ativos e $66,2 \%$, como mais ativos. (MAZO, 2003). De um total de 875 idosos avaliados acima de 60 anos de idade, $45 \%$ foram considerados menos ativos e $55 \%$, mais ativos. (BENEDETTI, 2004; BINOTO BORGATTO; FARIAS, 2010). Portanto, as prevalências são maiores para idosos mais ativos, tendo sido o valor de prevalência maior $(81,81 \%)$ nesta pesquisa, quando comparado com resultados anteriormente citados. Diante disso, pode-se inferir que os idosos que participam de grupos de convivência evidenciam, em sua maioria, bons níveis de atividade física.

Ao serem observados os diferentes domínios (vigoroso e moderado), constatou-se que há predominância das atividades físicas de lazer e recreação (33\% e 38\%) sobre os demais domínios. Em idosos norte-americanos, conforme estudos de Yusut et al. (1996) e Binoto, Borgatto e Farias (2010), foi verificado que $37 \%$ dos homens e $24 \%$ das mulheres eram mais ativos no lazer, quando reportado esse domínio especificamente.

Já em estudos realizados com idosas participantes de grupos de convivência, os maiores percentuais foram encontrados para atividades físicas no âmbito doméstico. De acordo com os estudos realizados por Mazo (2003), bem como por Binoto, Borgatto e Farias (2010), a prevalência de atividades físicas no âmbito doméstico foi de 40\%, enquanto Tribess (2006) e Binoto, Borgatto e Farias (2010) encontraram uma prevalência de $54,6 \%$ para esse domínio. Já para a presente pesquisa, houve a identificação de um percentual menor para atividades físicas no âmbito doméstico, encontrando-se $21 \%$.

O trabalho é o domínio que revela o menor índice de pessoas ativas (4\%), concordando com Borges et al. (2008), que analisaram uma amostra de idosos em Centros de Saúde (CS) em Florianópolis. Tais circunstâncias podem ser explicadas por alguns fatores: a maioria dos idosos entrevistados $(71,3 \%)$ não trabalha fora de casa; entre os que trabalham, a maior parte corresponde a voluntários que atuam de forma esporádica (uma ou duas vezes por semana), e os trabalhos rea- 
lizados são administrativos ou de apoio a grupos solidários, como igrejas, sem demanda significativa de atividade física.

Camarano (2001) demonstrou uma tendência de decréscimo na participação de idosos como força de trabalho no Brasil, bem como um número maior de homens trabalhando depois dos 60 anos, em comparação com as mulheres. Isso explica a baixa taxa de entrevistados que trabalham e ainda menor taxa dos que são fisicamente ativos durante $o$ trabalho, visto o grande percentual de mulheres no estudo.

No transporte, constatou-se que a maioria dos idosos não faz atividade física como meio de locomoção, tendo esse domínio obtido apenas $4 \%$. Apesar de os idosos caminharem como forma de deslocamento para atender as suas necessidades diárias, como ir ao supermercado, à feira, aos grupos de convivência etc., essas caminhadas, geralmente, são de curto período, não ultrapassando os 10 minutos necessários para serem classificadas como atividades físicas pelo IPAq. Resultados similares, principalmente em relação ao sexo feminino, foram encontrados em um estudo realizado em São Paulo. (SALVADOR et al., 2009).

A aderência está relacionada à percepção da importância da atividade física, que, consequentemente, influencia na frequência, na duração das sessões e no tempo de adesão aos exercícios físicos. (ALONSO; SANTOS; FIGUEIRA, 2006). Assim, o entendimento da devida importância da atividade física parece ser um fator de destaque que influencia os idosos na adesão às atividades físicas.
Considerando que os idosos, com o aumento da idade, apresentam uma tendência a diminuir o tempo semanal dedicado à prática de atividades físicas, principalmente os que já possuem patologias como hipertensão arterial e diabetes, sugere-se que a atenção em relação às estratégias de intervenção oferecidas pelos programas de atividades físicas seja voltada aos idosos com idades mais avançadas e à prevenção de doenças. Além disso, recomenda-se o investimento na sensibilização dos idosos quanto à importância da prática de atividades físicas regulares.

\section{Conclusão}

Os idosos de ambos os sexos que aceitaram participar deste estudo, no que se refere à prática de atividade física habitual realizada, em sua maioria, foram considerados mais ativos. $\mathrm{Na}$ contribuição dos diferentes domínios da atividade física, no dispêndio total em minutos por semana de atividades físicas, destacam-se com maior prevalência as atividades de lazer e/ou recreação, seguidas de atividades em casa. Porém, nas atividades laborais e de transporte, os idosos mostram-se menos ativos.

Sugere-se que mais estudos sejam efetuados com esse mesmo enfoque, a fim de verificar, no longo prazo, as principais barreiras e os motivos de não adesão aos programas de atividades físicas, assim como um acompanhamento periódico dos níveis de atividades físicas dessa população. 


\section{Profile of physical activity levels in elderly hypertensives and diabetics}

\section{Abstract}

The study aimed to verify the level of habitual physical activity in a group of elderly community. It is a cross-sectional descriptive and the non-probability sample consisted of 1919 elderly females aged more than 60 years $(68,73+6,99$ years $)$. For data collection, it was used a questionnaire with information related to satisfaction with health, disease and medication use and the International Physical Activity Questionnaire (IPAq) - long form, adapted for the elderly. The time of practice (full years) was informed through individual interviews. Data were analyzed using the statistical program SPSS 17.0, with descriptive analysis. Regarding the practice of physical activity, the elderly, most (71\%). In the contribution of different domains of physical activity in the total expenditure in minutes per week of physical activity, the most prevalent domain were the leisure activities and recreation (38\% and 33\%) respectively and activities at home (21\%). Therefore, older participants showed up assets, primarily in leisure / recreation, followed by activities at home, and less active in the field work and transport. It is suggested that further studies be conducted with this same approach in order to determine, in the long term, levels of physical activity in this population, such as periodic monitoring. Keywords: Elderly. Physical activity. Prevalence. Hypertension. Diabetes

\section{Agradecimentos}

Em especial, a Letícia Teixeira, Claudia Bucker e Joceléia Graeff Guillioni, pela contribuição no estudo.

\section{Referências}

ALONSO, D. O.; SANTOS, N. B; FIGUEIRA JR., A. J. The meaning of physical activity for the elderly: implications on adherence. Medicine \& Science in Sports \& Exercise, v. 38, n. 5, p. 42-50, 2006.

BENEDETTI, T. B. Atividade física: uma perspectiva de promoção da saúde do idoso no município de Florianópolis. 2004. 220 f. Tese (Doutorado em Enfermagem) - Faculdade de Enfermagem, Universidade Federal de Santa Catarina, Florianópolis, 2004.

BINOTO, M. A.; BORGATTO, A. F.; FARIAS, S. F. Nível de atividade física: questionário internacional de atividades físicas e tempo de prática em mulheres idosas. Revista Brasileira de Geriatria e Gerontologia, Rio de Janeiro, v. 13, n. 3, p. 425-434, 2010.

BORGES, L. J. et al. Exercício físico, déficits cognitivos e aptidão funcional de idosos usuários dos centros de saúde de Florianópolis. Revista Brasileira de Atividade Física \& Saúde, Florianópolis, v. 13, n. 3, p. 167-177, 2008.

CAMARANO, A. A. O idoso brasileiro no mercado de trabalho. In: CONGRESO NACIONAL DE ESTUDIOS DEL TRABAJO, 1, 2001, Buenos Aires. Anais... Buenos Aires: ASET, 2001.

CARVAlHO, J. A. M; GARCIA, R. A. The aging process in the Brazilian population: a demographic approach. Caderno de Saúde Pública, Rio de Janeiro, v. 19, n. 3, p. 725-733, 2003.

CASPERSEN, C. J.; POWELL, K. E.; CHRISTENSON, G. M. Physical activity, exercise and physical fitness: definitions and distinctions for health-related research. Public Health Reports, n. 2, p. 126-131, 2010.

DUARTE, R. et al. Recomendações da sociedade portuguesa de diabetologia para o tratamento da hiperglicemia e factores de risco na diabetes tipo 2. Revista Portuguesa de Diabetes, v. 2, n. 4, suppl, p. 5-18, 2007. 
FESKANICH, D.; WILLETT, W.; COLDITZ, G. Walking and leisure-time activity and risk of hip fracture in postmenopausal women. Jama, v. 288, n. 18, p. 2300-2306, 2002.

INSTITUTO BRASILEIRO DE GEOGRAFIA E ESTATÍSTICA (IBGE). Perfil dos idosos responsáveis pelos domicúlios no Brasil. 2002. Disponível em: <http://www.ibge.gov.br/ home/presidencia/noticias/25072002pidoso. pdf>. Acesso em: 14 out. 2008.

LEE, I. M.; SKERRETT, P. J. Physical activity and allcause mortality: what is the doseresponse relation? Medicine and Science in Sports and Exercise, v. 33, n. 6, p. 459-471, 2001.

MATSUDO, S. et al. Questionário Internacional de Atividade Física (IPAQ): estudo de validade e reprodutibilidade no Brasil. Revista Atividade Física \& Saúde, Florianópolis, v. 6, n. 2 , p. 5-18, 2001.

MAZO, G. Z. Atividade física e qualidade de vida de mulheres idosas. 2003. Tese (Doutorado em Educação Física) - Faculdade de Ciências de Desportos e de Educação Física, Universidade do Porto, Portugal. 2003.

OKUMA, S. S. O idoso e a atividade física. Campinas, São Paulo: Papirus, 2002, p. 39-40.

OLIVEIRA, S. M. J. V. et al. Hipertensão arterial referida em mulheres idosas: prevalência e fatores associados. Texto \& Contexto, Florianópolis, v. 17, n. 2, p. 241-249, 2008.

PAFFENBARGER, R. S. Some interrelations of physical activity, physiological fitness, health, and longevity. In: BOUCHARD, C.; SHEPARD, R. J.; STEPHENS, T. (Org.). Physical activity, fitness and health: international preceedings and consensua statement. Champaign, England: Humam Kinetcs Publishers, 1994. p. 119-133.

PAPALIA, D. E.; OLDS, S. W. Desenvolvimento humano. Trad. de Daniel Bueno. 7. ed. Porto Alegre: Artmed, 2000.
PATE, R. R. et al. Physical activity and public health: a recommendation from the Centers for Disease Control and Prevention and the American College of Sports Medicine. Journal of the American Geriatrics Society, v. 273, n. 5, p. 402-407, 1995.

Programa Nacional de Prevenção e Controlo da Diabetes PNPCD. Revista Portuguesa de Diabetes, v. 2, n. 4, p. 5-13, 2007.

RÊGO, A; et al. Pressão arterial após programa de exercício físico supervisionado em mulheres idosas hipertensas. Revista Brasileira de Medicina do Esporte, São Paulo, v. 17, n. 5, p. 300-304, 2011.

SALVADOR, E. P.; et al. A prática de caminhada como forma de deslocamento e sua associação com a percepção do ambiente em idosos. Revista Brasileira de Atividade Física \& Saúde, Florianópolis, v. 14, n. 3, p. 197-205, 2009.

SILVA, C.; et al. Adolescentes com Diabetes Mellitus Tipo 1: Adesão Insulinoterapia e Controlo Metabólico. Revista Portuguesa de Diabetes. v. 4, n. 1, 2009, p. 4-10.

SPIRDUSO, W. W. Dimensões físicas do envelhecimento. Barueri, São Paulo: Manole, 2005, p. 54-56.

SHEPHARD, R. J. Exercício e envelhecimento. Revista Brasileira de Ciência e Movimento, Brasília, v. 5, n. 4, p. 49-56, 1991.

TINETTI, M. E. Factors associated wich serious injury during falls by ambulatory nursing home residents. Journal of the American Geriatrics Society, v. 35, n. 10, p. 644-648, 1987.

TRIBESS, S. Percepção da imagem corporal e fatores relacionados à saúde em idosas. 2006. 112 f. Dissertação (Mestrado em Educação Física) - Faculdade de Educação Física, Universidade Federal de Santa Catarina, Florianópolis. 2006.

YUSUT, H. R. et al. Leisuretime physical activity among older adults. Archives of Internal Medicine, v. 156, n. 2, p. 1321-1326, 1996. 